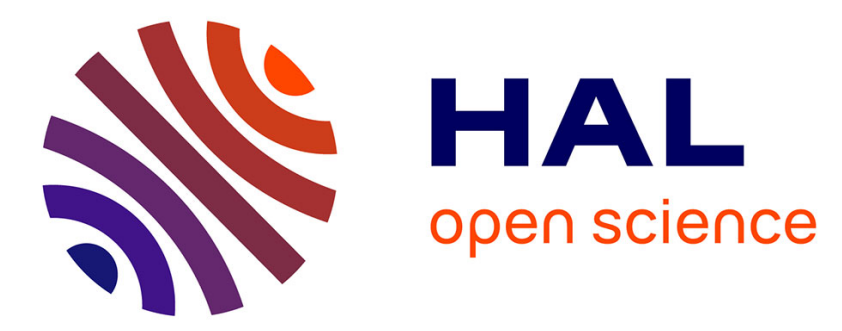

\title{
A self-healing control method for satellite attitude tracking based on simultaneous fault estimation and control design
}

Meng Zhou, Zhenhua Wang, Didier Theilliol, Yi Shen, Mickael Rodrigues

\section{- To cite this version:}

Meng Zhou, Zhenhua Wang, Didier Theilliol, Yi Shen, Mickael Rodrigues. A self-healing control method for satellite attitude tracking based on simultaneous fault estimation and control design. 3rd Conference on Control and Fault-Tolerant Systems, SysTol 2016, Sep 2016, Barcelone, Spain. hal01359731

\section{HAL Id: hal-01359731 \\ https://hal.science/hal-01359731}

Submitted on 3 Sep 2016

HAL is a multi-disciplinary open access archive for the deposit and dissemination of scientific research documents, whether they are published or not. The documents may come from teaching and research institutions in France or abroad, or from public or private research centers.
L'archive ouverte pluridisciplinaire HAL, est destinée au dépôt et à la diffusion de documents scientifiques de niveau recherche, publiés ou non, émanant des établissements d'enseignement et de recherche français ou étrangers, des laboratoires publics ou privés. 


\title{
A self-healing control method for satellite attitude tracking based on simultaneous fault estimation and control design
}

\author{
Meng Zhou ${ }^{1}$, Zhenhua Wang ${ }^{1}$, Didier Theilliol ${ }^{2}$, Yi Shen ${ }^{1}$, Mickael Rodrigues ${ }^{3}$
}

\begin{abstract}
This paper proposes a novel self-healing control method for satellite attitude tracking based on simultaneous fault estimation and control design. The proposed method integrates the fault estimation and fault-tolerant control units in a dynamic system, which is less complex and more reliable than the separately designed self-healing architectures. In this paper, the model reference approach is used to obtain a tracking error dynamic equation. Following this, an augmented error system is constructed by taking the fault as an auxiliary vector. Based on the augmented error system, a fault estimator/controller is designed to achieve robust fault-tolerant control and robust fault estimation simultaneously. The design conditions for the proposed fault estimator/controller are transformed as a set of linear matrix inequalities, which can be easily solved. Finally, numerical simulation results are given to demonstrate the effectiveness of proposed method.
\end{abstract}

\section{INTRODUCTION}

Although the components in the satellite are designed and selected to be highly reliable, failures cannot be completely avoided in the orbit operation. Therefore, it is important to take necessary measures to guarantee the performance and safety of satellite. During the past decades, many related methods have been proposed to deal with the faults and ensure the satellite attitude control performance. [1] proposes an observer-based fault diagnosis method and a backstepping-based fault tolerant control law to deal with the loss of effectiveness fault. In [2], an adaptive failure compensation scheme is first developed to guarantee microsatellite system stability and asymptotic tracking properties, and then an adaptive failure identification scheme is designed by using multiple estimators. [3] proposes an indirect approach for fault diagnosis and fault-tolerant control in the satellite attitude control system with sampled-data measurements. In [4], a trajectory planning/re-planning method based is designed on attainable efforts analysis is applied for satellite system in rendezvous mission. However, it is noted that most of the existing results consider the fault diagnosis module and fault-tolerant control scheme sperately. This may bring time delay in real application [5]. In order to deal with the

\footnotetext{
*This work was partially supported by National Natural Science Foundation of China (Grant No. 61273162, 61403104) and the Fundamental Research Funds for the Central Universities under Grant HIT.KLOF.2015.076.

${ }^{1}$ Meng Zhou, Zhenhua Wang and Yi Shen are with the Deptartment of Control Science and Engineering, Harbin Institute of Technology, 150001 Harbin, China zhenhua.wangehit. edu. cn

2 Didier Theilliol is with the Centre de Recherche en Automatique de Nancy, Université de Lorraine, CNRS UMR 7039, F-54506 Vandoeuvreles-Nancy, France didier.theilliol@univ-lorraine.fr

${ }^{3}$ Mickael Rodrigues is with the Laboratoire d'Automatique et Génie des Procédés, Université de Lyon, Lyon, F-69003, France mickael.rodrigues@univ-lyonl. fr
}

drawbacks, Liu et al. investigated integrated design of fault diagnosis and fault-tolerant control [6], [7]. However, the fault diagnosis module and fault-tolerant control module in the literatures are still designed separately.

Recently, the concept of self-healing control has been proposed to include the fault diagnosis and fault-tolerant control methods in a unified frame [8], [9]. Self-healing control methods are a class of control architectures which make the system counteract faults actively and maintain a certain level of control performance [8]. Simultaneous Fault Detection and Control (SFDC) provides an effetive solution to self-healing control design and has attracted much attention in recent years [10], [11], [12], [13], [14]. The basic idea of SFDC is to integrate the control module and diagnostic module into one system unit such that control objective and fault detection objective can be satisfied simultaneously [15]. In [11], the SFDC problem is formulated as a mixed optimization problem and its solution is presented in terms of two coupled Riccati equations. A dynamic observer is presented to handle the SFDC problem for switched system in [12]. In [13] a robust SFDC approach is applied to the nonlinear longitudinal dynamics of Boeing 747-100/200 aircraft. The SFDC problem is investigated in [14] for continuoustime linear system and the proposed method is applied to a satellite system. However, the output of satellite system is only regulated to zero by the the proposed method in [14], while in most situations, satellite controller must have the capacity to regulate the output asymptotically track a reference attitude, especially for an on-orbit satellite. Besides, it should be noted that only a few results considers the problem of simultaneous fault estimation and fault tolerant control. In [16], an integrated fault estimation and control system reconfiguration is designed, in which the fault estimator is based on a modified reduced-/full- order unknown input observer and the fault tolerant controller is based on a sliding mode control using state/output feedback. In this paper, we mainly focuses on the self-healing attitude tracking control design based on simultaneous fault estimation and control method for the linear satellite attitude tracking system.

This paper proposes a novel self-healing control method for satellite attitude tracking control. The main contribution of this paper lies in two-fold. First, the fault estimation objective and fault-tolerant attitude tracking control performance are satisfied simultaneously by designing a robust fault estimator/controller for an error system between the satellite system and reference model. Furthermore, the proposed fault estimator/controller design conditions are transformed into a set of Linear Matrix Inequalities (LMIs). 


\section{PROBLEM FORMULATION}

\section{A. Satellite model formulation}

Based on the Euler moment equation, the attitude dynamic of a rigid satellite is obtaind as

$$
J \dot{\omega}=-\omega^{\times} J \omega+T_{u}+T_{v}
$$

where $J \in \mathbb{R}^{3 \times 3}$ denotes the momentum inertia matrix, $\omega=\left[\omega_{x}, \omega_{y}, \omega_{z}\right]^{T}$ represents the angular velocity of the body frame with respect to the inertial frame, $T_{u}=$ $\left[T_{u x}, T_{u y}, T_{u z}\right]^{T}$ denotes the torques provided by the actuator and $T_{v}=\left[T_{v x}, T_{v y}, T_{v z}\right]^{T}$ represents the external disturbance torques. $\omega^{\times}$denotes a skew-symmetric matrix defined as

$$
\omega^{\times}=\left[\begin{array}{ccc}
0 & -\omega_{z} & \omega_{y} \\
\omega_{z} & 0 & -\omega_{x} \\
-\omega_{y} & \omega_{x} & 0
\end{array}\right]
$$

In this paper, it is assumed that $J=\operatorname{diag}\left(\left[J_{x}, J_{y}, J_{z}\right]\right)$, where $J_{x}, J_{y}, J_{z}$ are the principal moments of inertia. Then the system (1) can be written as

$$
\left\{\begin{array}{l}
J_{x} \dot{\omega}_{x}-\left(J_{y}-J_{z}\right) \omega_{y} \omega_{z}=T_{u x}+T_{v x} \\
J_{y} \dot{\omega}_{y}-\left(J_{z}-J_{x}\right) \omega_{z} \omega_{x}=T_{u y}+T_{v y} \\
J_{z} \dot{\omega}_{z}-\left(J_{x}-J_{y}\right) \omega_{x} \omega_{y}=T_{u z}+T_{v z}
\end{array}\right.
$$

Under a small attitude angle condition [17], [18], the attitude kinematic equation of the satellite is expressed as

$$
\left\{\begin{array}{l}
\omega_{x}=\dot{\phi}-\omega_{0} \psi \\
\omega_{y}=\dot{\theta}-\omega_{0} \\
\omega_{z}=\dot{\psi}+\omega_{0} \phi
\end{array}\right.
$$

where $\omega_{0}$ is the orbital rate of the satellite and $\phi, \theta, \psi$ are roll, pitch and yaw angle, respectively.

The external disturbance $T_{v}$ mainly consisted with gravity gradient torque $T_{g}$ and the other environmental disturbance $T_{d}$. The gravity-gradient torque is approximated by

$$
\left\{\begin{array}{l}
T_{g x}=3 \omega_{0}^{2}\left(J_{z}-J_{y}\right) \phi \\
T_{g y}=3 \omega_{0}^{2}\left(J_{z}-J_{x}\right) \theta \\
T_{g z}=0
\end{array}\right.
$$

Then substitute (4) and (5) into (3), then (3) can be approximated by

$$
\left\{\begin{array}{l}
J_{x} \ddot{\phi}-\omega_{0}\left(J_{x}-J_{y}+J_{z}\right) \dot{\psi}+4 \omega_{0}^{2}\left(J_{y}-J_{z}\right) \phi=T_{u x}+T_{d x} \\
J_{y} \ddot{\theta}+3 \omega_{0}^{2}\left(J_{x}-J_{z}\right) \theta=T_{u y}+T_{d y} \\
J_{z} \ddot{\psi}+\omega_{0}\left(J_{x}-J_{y}+J_{z}\right) \dot{\phi}+\omega_{0}^{2}\left(J_{y}-J_{x}\right) \psi=T_{u z}+T_{d z}
\end{array}\right.
$$

Define $x=\left[\begin{array}{llllll}\phi & \theta & \psi & \dot{\phi} & \dot{\theta} & \dot{\psi}\end{array}\right]^{T}$ as the state vector and consider the additive actuator fault $T_{f}$, the system (6) is rewritten as

$$
\dot{x}(t)=A_{c} x(t)+B_{c}\left(T_{u}(t)+T_{f}(t)\right)+B_{c w} T_{d}(t)
$$

where the system matrices are

$$
A_{c}=\left[\begin{array}{cc}
0 & I_{3} \\
-M^{-1} K & -M^{-1} D
\end{array}\right]
$$

$$
B_{c}=\left[\begin{array}{c}
0 \\
M^{-1} I_{3}
\end{array}\right], B_{c w}=\left[\begin{array}{c}
0 \\
M^{-1} I_{3}
\end{array}\right]
$$

with

$$
\begin{gathered}
M=\left[\begin{array}{ccc}
J_{x} & 0 & 0 \\
0 & J_{y} & 0 \\
0 & 0 & J_{z}
\end{array}\right] \\
K=\left[\begin{array}{ccc}
4 \omega_{0}^{2}\left(J_{y}-J_{z}\right) & 0 & 0 \\
0 & 3 \omega_{0}^{2}\left(J_{x}-J_{z}\right) & 0 \\
0 & 0 & \omega_{0}^{2}\left(J_{y}-J_{x}\right)
\end{array}\right] \\
D=\left[\begin{array}{ccc}
0 & 0 & -\omega_{0}\left(J_{x}+J_{z}-J_{y}\right) \\
0 & 0 & 0 \\
\omega_{0}\left(J_{x}+J_{z}-J_{y}\right) & 0 & 0
\end{array}\right]
\end{gathered}
$$

In this paper, it is assumed that only the Euler angles are measured. i.e.

$$
y(t)=C x(t)
$$

where $C=\left[\begin{array}{ll}I_{3} & 0\end{array}\right]$.

In this paper, the continuous-time model (7) is approximated by using the Euler approximation method. For a given sampling period $t_{s}$, the discrete-time approximation of the satellite is obtained as follows

$$
\left\{\begin{array}{l}
x(k+1)=A x(k)+B T_{u}(k)+B_{f} T_{f}(k)+B_{w} T_{d}(k) \\
y(k)=C x(k)
\end{array}\right.
$$

where $A=I_{6}+A_{c} t_{s}, B=B_{f}=B_{c} t_{s}, B_{w}=B_{c w} t_{s}$.

\section{B. Attitude tracking error model}

In this paper, the model reference method is used to design tracking controller for system (9). The reference model is given by

$$
\left\{\begin{array}{l}
x_{r}(k+1)=A x_{r}(k)+B T_{u r}(k) \\
y_{r}(k)=C x_{r}(k)
\end{array}\right.
$$

where $x_{r} \in \mathbb{R}^{6}, T_{u r} \in \mathbb{R}^{3}$, and $y_{r} \in \mathbb{R}^{3}$ are the state, control input and output vectors of the reference model, respectively. The output of the reference system is the desired trajectory. Therefore, the following control law is presented for the reference model (10)

$$
T_{u r}(k)=K_{x} x_{r}(k)+K_{r} r(k)
$$

where $K_{x} \in \mathbb{R}^{3 \times 6}$ is the state feedback control matrix which is designed such that $A+B K_{x}$ have to be stable and $K_{r} \in$ $\mathbb{R}^{3}$ is the feedforward control matrix which regulates the output of the reference model $y_{r}(k)$ to track the reference attitude $r(k)$ [9].

To address the attitude tracking problem, attitude tracking error is defined as follows

$$
\Delta x(k)=x(k)-x_{r}(k)
$$

Then, by subtracting (9) from (10), the error dynamic equation is obtained as

$$
\left\{\begin{aligned}
& \Delta x(k+1)= A \Delta x(k)+B \Delta T_{u}(k)+B_{f} T_{f}(k) \\
&+B_{w} T_{d}(k) \\
& \Delta y(k)=C \Delta x(k)
\end{aligned}\right.
$$


where

$$
\begin{gathered}
\Delta T_{u}(k)=T_{u}(k)-T_{u r}(k) \\
\Delta y(k)=y(k)-y_{r}(k)
\end{gathered}
$$

It can be seen that if a suitable controller $\Delta T_{u}(k)$ is designed such that the attitude error converge asymptotically to zero, then the attitude of the satellite will track the desired attitude.

\section{The SFEC problem}

Motivated by [19], [20], this paper assumes that the fault dynamics is described as

$$
T_{f}(k+1)=A_{f} T_{f}(k)+\Delta T_{f}(k)
$$

where $\Delta T_{f}(k)$ is the error of fault model between $\mathrm{t}$ wo consecutive time interval, $A_{f}$ is defined by $A_{f}=$ $\operatorname{diag}\left[\left(A_{f 1}, A_{f 2}, A_{f 3}\right]\right)$. Note that $A_{f}$ represents the additional degree of freedom in SFEC design which is obtained to add another state to the new state equations. Besides, $A_{f}$ should be fixed between $[0 \cdots 1]$.

In order to faciliate the SFEC design, the state augmentation approach is used in this paper. The actuator fault $T_{f}(k)$ is considered as an auxiliary state vector, i.e.

$$
\eta(k)=\left[\begin{array}{ll}
\Delta x^{T}(k) & T_{f}^{T}(k)
\end{array}\right]^{T}
$$

Using (13) and (16), an augmented system is obtained as

$$
\left\{\begin{array}{l}
\eta(k+1)=\bar{A} \eta(k)+\bar{B} \Delta T_{u}(k)+\bar{B}_{w} w(k) \\
\Delta y(k)=\bar{C} \eta(k)
\end{array}\right.
$$

where

$$
\begin{gathered}
\bar{A}=\left[\begin{array}{cc}
A & B_{f} \\
0 & A_{f}
\end{array}\right], \bar{B}=\left[\begin{array}{c}
B \\
0
\end{array}\right], w(k)=\left[\begin{array}{c}
T_{d}(k) \\
\Delta T_{f}(k)
\end{array}\right], \\
\bar{B}_{w}=\left[\begin{array}{cc}
B_{d} & 0 \\
0 & I_{3}
\end{array}\right], \bar{C}=\left[\begin{array}{ll}
C & 0
\end{array}\right]
\end{gathered}
$$

Based on the augmented system (18), the proposed fault estimator/controller is given as follows

$$
\left\{\begin{array}{l}
\hat{\eta}(k+1)=A_{F} \hat{\eta}(k)+B_{F} \Delta y(k) \\
\Delta T_{u}(k)=K_{F} \hat{\eta}(k) \\
\hat{T}_{f}(k)=D_{F} \hat{\eta}(k)
\end{array}\right.
$$

where $\hat{\eta}(k) \in \mathbb{R}^{9}$ is the state vector of the proposed fault estimator/controller, and $A_{F} \in \mathbb{R}^{9 \times 9}, B_{F} \in \mathbb{R}^{9 \times 3}, K_{F} \in$ $\mathbb{R}^{3 \times 9}, D_{F} \in \mathbb{R}^{3 \times 9}$ are the parameter matrices to be designed.

In this paper, the SFEC design is transformed into a multiple-objective $H_{\infty}$ optimization problem stated as follows.

SFEC design: Given two scalars $\gamma_{y}>0$ and $\gamma_{e}>0$, determine the matrices $A_{F}, B_{F}, K_{F}, D_{F}$ in (19) such that

(i) The error system (13) is asymptotically stable such that the output of the faulty system can track the reference trajectory.

(ii) The tracking error of controlled output $\Delta y(k)$ is robust to disturbance $w(k)$, i.e.

$$
\|\Delta y\|_{2}<\gamma_{y}\|w\|_{2}
$$

(iii) The error of fault estimation $e_{f}(k)$ is robust to disturbance $w(k)$, i.e.

$$
\left\|e_{f}\right\|_{2}<\gamma_{e}\|w\|_{2}
$$

where $e_{f}(k)=T_{f}(k)-\hat{T}_{f}(k)$ denotes the fault estimation error.

\section{SIMULTANEOUS FAULT ESTIMATION AND CONTROL DESIGN}

In this section, the parameter matrices $A_{F}, B_{F}, K_{F}, D_{F}$ in the fault estimator/controller (19) are designed to safisfy the conditions (i)-(iii).

Combing (18) and (19), the overall system is expressed as

$$
\left\{\begin{array}{l}
\eta(k+1)=\bar{A} \eta(k)+\bar{B} K_{F} \hat{\eta}(k)+\bar{B}_{w} w(k) \\
\hat{\eta}(k+1)=A_{F} \hat{\eta}(k)+B_{F} \bar{C} \eta(k) \\
\Delta y(k)=\bar{C} \eta(k) \\
e_{f}(k)=C_{f} \eta(k)-D_{F} \hat{\eta}(k)
\end{array}\right.
$$

Herein, the following relation is used

$$
T_{f}(k)=C_{f} \eta(k)
$$

where $C_{f}=\left[\begin{array}{ll}0 & I_{3}\end{array}\right]$.

For the sake of brevity, we define

$$
\xi(k)=\left[\begin{array}{ll}
\eta(k) & \hat{\eta}(k)
\end{array}\right]^{T}
$$

The overall system (22) is formulated as the following two subsystems

$$
\left\{\begin{array}{l}
\xi(k+1)=\tilde{A} \xi(k)+\tilde{B} w(k) \\
\Delta y(k)=\tilde{C}_{y} \xi(k) \\
e_{f}(k)=\tilde{C}_{e} \xi(k)
\end{array}\right.
$$

where

$$
\begin{aligned}
& \tilde{A}=\left[\begin{array}{cc}
\bar{A} & \bar{B} K_{F} \\
B_{F} \bar{C} & A_{F}
\end{array}\right], \tilde{B}=\left[\begin{array}{c}
\bar{B}_{w} \\
0
\end{array}\right], \\
& \tilde{C}_{y}=\left[\begin{array}{ll}
\bar{C} & 0
\end{array}\right], \tilde{C}_{e}=\left[\begin{array}{ll}
C_{f} & -D_{F}
\end{array}\right]
\end{aligned}
$$

Based on (25), the following theorem is proposed to design the parameter matrices of the estimator/controller (19) to satisfy the two above optimal problems.

Theorem 1. Given $\gamma_{y}>0$ and $\gamma_{e}>0$, there exists positivedefinite matrices $X=X^{T} \in \mathbb{R}^{9 \times 9}, Y=Y^{T} \in \mathbb{R}^{9 \times 9}$ and matrices $\hat{A} \in \mathbb{R}^{9 \times 9}, \hat{B} \in \mathbb{R}^{9 \times 3}, \hat{C} \in \mathbb{R}^{3 \times 9}, \hat{D} \in \mathbb{R}^{3 \times 9}$ such that the following LMIs hold:

$$
\begin{aligned}
& {\left[\begin{array}{cccccc}
-X & * & * & * & * & * \\
-I_{9} & -Y & * & * & * & * \\
0 & 0 & -\gamma_{y}^{2} I_{6} & * & * & * \\
\bar{C} X & \bar{C} & 0 & -I_{3} & * & * \\
\bar{A} X+\bar{B} \hat{C} & \bar{A} & \bar{B}_{w} & 0 & -X & * \\
\hat{A} & Y \bar{A}+\hat{B} \bar{C} & Y \bar{B}_{w} & 0 & -I_{9} & -Y
\end{array}\right]<0} \\
& {\left[\begin{array}{cccccc}
-X & * & * & * & * & * \\
-I_{9} & -Y & * & * & * & * \\
0 & 0 & -\gamma_{e}^{2} I_{6} & * & * & * \\
C_{f} X-\hat{D} & C_{f} & 0 & -I_{3} & * & * \\
\bar{A} X+\bar{B} \hat{C} & \bar{A} & \bar{B}_{w} & 0 & -X & * \\
\hat{A} & Y \bar{A}+\hat{B} \bar{C} & Y \bar{B}_{w} & 0 & -I_{9} & -Y
\end{array}\right]<0}
\end{aligned}
$$


then the closed-loop system (22) is asymptotic stable and the designed estimator/controller (19) achieves the attitude tracking control performance (ii) and fault estimation objective (iii) simultaneously. Moreover, the parameter matrices $A_{F}, B_{F}, K_{F}$ and $D_{F}$ can be determined by

$$
\begin{gathered}
A_{F}=N^{-1}(\hat{A}-\hat{B} \bar{C} X-Y \bar{B} \hat{C}-Y \bar{A} X) M^{-T} \\
B_{F}=N^{-1} \hat{B}, K_{F}=\hat{B} M^{-T}, D_{F}=\hat{D} M^{-T}
\end{gathered}
$$

where

$$
\begin{gathered}
\hat{A}=Y \bar{A} X+N B_{F} \bar{C} X+Y \bar{B} K_{F} M^{T}+N A_{F} M^{T}, \\
\hat{B}=N B_{F}, \hat{C}=K_{F} M^{T}, \hat{D}=D_{F} M^{T}
\end{gathered}
$$

with $M \in \mathbb{R}^{9 \times 9}$ and $N \in \mathbb{R}^{9 \times 9}$ solved from

$$
M N^{T}=I-X Y
$$

Proof. Applying the bounded real lemma [21] to system (25), it is known that conditions (i)-(iii) are satisfied if there exists a positive-definite matrix $P>0$ such that

$$
\left[\begin{array}{cc}
\tilde{A}^{T} P \tilde{A}-P+\tilde{C}_{y}^{T} \tilde{C}_{y} & \tilde{A}^{T} P \tilde{B} \\
\tilde{B}^{T} P \tilde{A} & \tilde{B}^{T} P \tilde{B}-\gamma_{y}^{2} I_{6}
\end{array}\right]<0
$$

and

$$
\left[\begin{array}{cc}
\tilde{A}^{T} P \tilde{A}-P+\tilde{C}_{e}^{T} \tilde{C}_{e} & \tilde{A}^{T} P \tilde{B} \\
\tilde{B}^{T} P \tilde{A} & \tilde{B}^{T} P \tilde{B}-\gamma_{e}^{2} I_{6}
\end{array}\right]<0
$$

hold.

We first show that (32) holds if (27) is fulfilled. Using the Schur complement lemma [22], (32) is equivalent to

$$
\left[\begin{array}{cccc}
-P & 0 & \tilde{C}_{y}^{T} & \tilde{A}^{T} P \\
0 & -\gamma_{y}^{2} I_{6} & 0 & \tilde{B}^{T} P \\
\tilde{C}_{y} & 0 & -I_{3} & 0 \\
P \tilde{A} & P \tilde{B} & 0 & -P
\end{array}\right]<0
$$

It should be noted that (34) is not a standard linear matrix inequality of the parameter matrices $A_{F}, B_{F}, K_{F}, D_{F}$. Therefore, further transformations are needed to make (34) solvable. To this end, according to [23], the matrices $P$ and $P^{-1}$ are partitioned as

$$
P=\left[\begin{array}{cc}
Y & N \\
N^{T} & R
\end{array}\right], P^{-1}=\left[\begin{array}{cc}
X & M \\
M^{T} & S
\end{array}\right]
$$

where $X$ and $Y$ are symmetric matrices. It is noted that the matrices $X, Y, M, N, R$ and $S$ should fulfill $P P^{-1}=I_{9}$, which implies that (31) holds.

Constructing two matrices $\Pi_{1}$ and $\Pi_{2}$ as

$$
\Pi_{1}=\left[\begin{array}{cc}
X & I_{9} \\
M^{T} & 0
\end{array}\right], \Pi_{2}=\left[\begin{array}{cc}
I_{9} & Y \\
0 & N^{T}
\end{array}\right]
$$

From (35) and (36), it can been easily derived that

$$
P \Pi_{1}=\Pi_{2}
$$

Pre- and post- multiplying (34) by $\operatorname{diag}\left(\Pi_{1}^{T}, I, I, \Pi_{1}^{T}\right)$ and $\operatorname{diag}\left(\Pi_{1}, I, I, \Pi_{1}\right)$, respectively, it comes

$$
\left[\begin{array}{cccc}
-\Pi_{1}^{T} P \Pi_{1} & 0 & \Pi_{1}^{T} \tilde{C}_{y}^{T} & \Pi_{1}^{T} \tilde{A}^{T} P \Pi_{1} \\
0 & -\gamma_{y}^{2} I_{6} & 0 & \tilde{B}^{T} P \Pi_{1} \\
\tilde{C}_{y} \Pi_{1} & 0 & -I_{3} & 0 \\
\Pi_{1}^{T} P \tilde{A} \Pi_{1} & \Pi_{1}^{T} P \tilde{B} & 0 & -\Pi_{1}^{T} P \Pi_{1}
\end{array}\right]<0
$$

Considering the definitions of $\tilde{A}, \tilde{B}, \tilde{C}_{y}$ in (26), and using the equation (37), it can be derived that

$$
\begin{gathered}
\Pi_{1}^{T} P \Pi_{1}=\left[\begin{array}{cc}
X & I_{9} \\
I_{9} & Y
\end{array}\right], \Pi_{1}^{T} P \tilde{A} \Pi_{1}=\left[\begin{array}{ll}
\mathcal{M}_{11} & \mathcal{M}_{12} \\
\mathcal{M}_{21} & \mathcal{M}_{22}
\end{array}\right], \\
\Pi_{1}^{T} P \tilde{B}=\left[\begin{array}{c}
\bar{B}_{w} \\
Y \bar{B}_{w}
\end{array}\right], \tilde{C}_{y} \Pi_{1}=\left[\begin{array}{ll}
\bar{C}_{z} X & \bar{C}_{z}
\end{array}\right]
\end{gathered}
$$

where

$$
\begin{gathered}
\mathcal{M}_{11}=\bar{A} X+\bar{B} K_{F} M^{T}, \mathcal{M}_{12}=\bar{A} \\
\mathcal{M}_{21}=Y \bar{A} X+N B_{F} \bar{C} X+Y \bar{B} K_{F} M^{T}+N A_{F} M^{T}, \\
\mathcal{M}_{22}=Y \bar{A}+N B_{F} \bar{C}
\end{gathered}
$$

Using the definitions of $\hat{A}, \hat{B}$ and $\hat{C}$ in (30), the term $\Pi_{1}^{T} P \tilde{A} \Pi_{1}$ is rewritten as

$$
\Pi_{1}^{T} P \tilde{A} \Pi_{1}=\left[\begin{array}{cc}
\bar{A} X+\bar{B} \hat{C} & \bar{A} \\
\hat{A} & Y \bar{A}+\hat{B} \bar{C}
\end{array}\right]
$$

Substituting (39),(40) into (38), the inequality (38) becomes (27). Therefore, it can be concluded that (27) is sufficient for the conditions (i) and (ii).

By using a similar procedure, it can also be shown that (28) is equivalent to the inequality (33). Herein, the details of this proof are omitted for the sake of brevity.

Once the matrices $\hat{A}, \hat{B}, \hat{C}, \hat{D}, M, N$ are determined, the matrices $A_{F}, B_{F}, K_{F}, D_{F}$ can be obtained by (29).

\section{SIMULATIONS}

In this section, numerical simulations are used to verify the effectiveness of the proposed method. Table 1 gives the satellite parameters, which are borrowed from [14].

TABLE 1

SATELLITE PARAMETERS

\begin{tabular}{c|c|c|c}
\hline$J_{x}$ & $12.49 \mathrm{~kg} \cdot \mathrm{m}^{2}$ & $\omega_{0}$ & $0.0011 \mathrm{rad} / \mathrm{s}$ \\
\hline$J_{y}$ & $13.85 \mathrm{~kg} \cdot \mathrm{m}^{2}$ & $A_{s}$ & $1.5 \times 10^{-4} \mathrm{Nm}$ \\
\hline$J_{z}$ & $15.75 \mathrm{~kg} \cdot \mathrm{m}^{2}$ & \multicolumn{3}{|c}{} \\
\hline
\end{tabular}

And the external disturbance is assumed to be as

$$
\left\{\begin{array}{l}
T_{d x}=A_{s}\left(3 \cos \omega_{0} k+1\right) \\
T_{d y}=A_{s}\left(3 \cos \omega_{0} k+1.5 \sin \omega_{0} k\right) \\
T_{d z}=A_{s}\left(3 \sin \omega_{0} k+1\right)
\end{array}\right.
$$

where $A_{s}$ is the amplitude of the disturbance.

Combined with the definition of the system matrices, it is easily to obtain that

$$
\begin{gathered}
A=\left[\begin{array}{cccccc}
1.0000 & 0 & 0 & 0.1000 & 0 & 0 \\
0 & 1.0000 & 0 & 0 & 0.1000 & 0 \\
0 & 0 & 1.0000 & 0 & 0 & 0.1000 \\
0 & 0 & 0 & 1.0000 & 0 & 0.0001 \\
0 & 0.00 & 0 & 0 & 1.0000 & 0 \\
0 & 0 & -0 & -0.0001 & 0 & 1.0000
\end{array}\right] \\
B=B_{w}=\left[\begin{array}{ccc}
0 & 0 & 0 \\
0 & 0 & 0 \\
0 & 0 & 0 \\
0.08 & 0 & 0 \\
0 & 0.0072 & 0 \\
0 & 0 & 0.0063
\end{array}\right]
\end{gathered}
$$


In the SFEC design, the fault system matrix is assumed as $A_{f}=0.99 I_{3}$ and the $H_{\infty}$ performance indexes are chosen as $\gamma_{y}=5, \gamma_{e}=5$. By solving the LMIs (28) and (27) in Theorem 1, the matrices in the proposed fault estimator/controller gain matrices are obtained as

$$
\begin{gathered}
A_{F}=\left[\begin{array}{ll}
A_{F 1} & A_{F 2}
\end{array}\right], B_{F}=\left[\begin{array}{l}
B_{F 1} \\
B_{F 2}
\end{array}\right] \\
K_{F}=\left[\begin{array}{ll}
K_{F 1} & K_{F 2}
\end{array}\right], D_{F}=\left[\begin{array}{l}
D_{F 1} \\
D_{F 2}
\end{array}\right]
\end{gathered}
$$

where

$$
\begin{aligned}
& A_{F 1}=\left[\begin{array}{ccccc}
0.988 & 0 & 0 & -0.0002 & -0 \\
0 & 0.9878 & 0 & -0 & -0.5490 \\
0 & -0 & 0.9876 & 0.5261 & -0 \\
-0 & -0 & 0.0086 & -0.8857 & 0 \\
0 & -0.0075 & -0 & -0 & -0.9154 \\
-0.0067 & -0 & -0 & -0 & 0 \\
0 & 0 & -0.0012 & 0.2688 & 0 \\
-0 & 0.0012 & 0 & -0 & 0.2955 \\
-0.0011 & 0 & -0 & 0 & -0
\end{array}\right] \\
& A_{F 2}=\left[\begin{array}{cccc}
-0.5675 & -0.0013 & 0 & 2.4931 \\
0 & 0 & -2.5646 & 0 \\
-0.0003 & 2.6519 & -0 & 0.0013 \\
0 & -7.8000 & 0 & 0.0001 \\
0 & 0 & -7.3184 & 0 \\
-0.9406 & 0.0001 & 0 & 6.9510 \\
0 & 0 & -0.9508 & 0 \\
0 & 0 & -0.9618 & 0 \\
-0.3193 & 0 & 0 & -0.9721
\end{array}\right] \\
& B_{F 1}=\left[\begin{array}{ccccc}
-49.885 & -0 & -0.0151 & -0.0326 & -0 \\
-0 & -48.78 & -0 & -0 & -223.562 \\
0.0125 & 0 & -47.1873 & 224.65 & -0
\end{array}\right]^{T} \\
& B_{F 2}=\left[\begin{array}{cccc}
-222.3986 & 0.0291 & -0 & -155.3724 \\
0 & 0 & 152.4019 & -0 \\
-0.0271 & -148.7478 & 0 & -0.0286
\end{array}\right]^{T} \\
& K_{F 1}=\left[\begin{array}{ccccc}
38.9880 & 0 & 0.0206 & 0.0004 & -0 \\
-0 & 39.5307 & 0 & -0 & -18.0183 \\
-0.02 & -0 & 40.184 & 18.1938 & -0
\end{array}\right] \\
& K_{F 2}=\left[\begin{array}{cccc}
-17.8742 & -0.0054 & 0 & 50.6313 \\
0 & -0 & -54.7466 & 0 \\
-0.0005 & 60.4595 & -0 & 0.0068
\end{array}\right] \\
& D_{F 1}=\left[\begin{array}{ccccc}
-39.007 & -0 & -0.0206 & -0.0006 & 0 \\
0 & -39.553 & -0 & -0 & 12.264 \\
0.0200 & 0 & -40.2117 & -12.1145 & 0
\end{array}\right]^{T} \\
& D_{F 2}=\left[\begin{array}{cccc}
12.3598 & 0.0016 & -0 & -7.6068 \\
-0 & 0 & 7.9253 & -0 \\
0.0001 & -8.3446 & 0 & -0.0018
\end{array}\right]^{T}
\end{aligned}
$$

In the simulation, the reference attitude is assumed as $r(k)=\left[\begin{array}{lll}0.01 & 0.02 & 0.05\end{array}\right]^{T}(\mathrm{rad})$. The matrices $K_{x}$ and $K_{r}$ are given as

$$
\begin{gathered}
K_{x}=\left[\begin{array}{cccccc}
-47.08 & 0 & 0 & -132.03 & 0 & -0.016 \\
0 & -52.21 & 0 & 0 & -146.41 & 0 \\
0 & 0 & -59.37 & 0.016 & 0 & -166.5
\end{array}\right] \\
K_{r}=\left[\begin{array}{cccc}
47.0812 & 0 & 0 \\
0 & 52.2078 & 0 \\
0 & 0 & 59.3699
\end{array}\right]
\end{gathered}
$$

In the simulation, the modelling uncertainty is considered as $\Delta A=10^{-3} A \sin (0.02 k)$. In order to verify the effectiveness of the proposed method, the commonly encountered fault scenarios are considered in the simulation. First, an abrupt fault scenario is simulated. In the simulation, it is assumed that two abrupt faults simultaneously occur at $k=$ 200 with the following characteristics

$$
T_{f}(k)=\left\{\begin{array}{lll}
{\left[\begin{array}{lll}
0 & 0 & 0
\end{array}\right]^{T}} & k<200 \\
{\left[\begin{array}{lll}
-0.5 & 1.2 & 0
\end{array}\right]^{T}} & 200 \leqslant k<600
\end{array}\right.
$$

The fault-tolerant control and fault estimation results are depicted in Fig. 1 and Fig. 2, respectively. From Fig. 1, it is seen that the desired attitude are followed with high accuracy even in the presence of actuator failure and external disturbances. Moreover, the last three states illustrate that the proposed fault estimator/controller ensure the whole system stabilized. Fig. 2 shows that the proposed fault estimator/controller provides quick and accurate fault estimations.

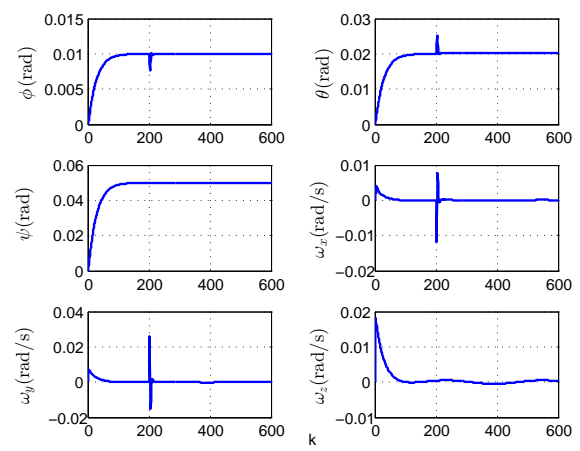

Fig. 1. Fault-tolerant control result in an abrupt actuator fault scenario

In the second scenario, it is assumed that a time-varying fault occurs with the following form

$$
T_{f}(k)= \begin{cases}{\left[\begin{array}{lll}
0 & 0 & 0
\end{array}\right]^{T}} & k<100 \\
{\left[\begin{array}{lll}
0 & 0.03(k-100) & 0
\end{array}\right]^{T}} & 100 \leqslant k<300 \\
{\left[\begin{array}{lll}
0 & 0.6-0.03(k-100) & 0
\end{array}\right]^{T}} & 300 \leqslant k<500 \\
{\left[\begin{array}{lll}
0 & 0 & 0
\end{array}\right]^{T}} & 500 \leqslant k<600\end{cases}
$$

The fault-tolerant control and fault estimation results are depicted in Fig. 3 and Fig. 4, respectively. It can be seen that the proposed method also guarantee the attitude tracing performance and robust fault estimation performance in the presence of time-varying fault. 


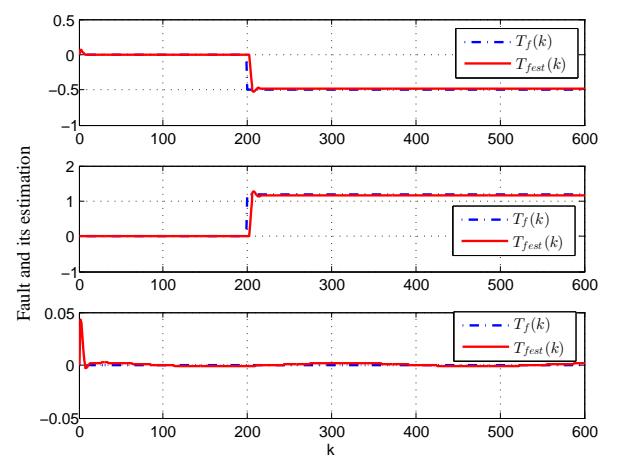

Fig. 2. Fault estimation result in an abrupt fault scenario
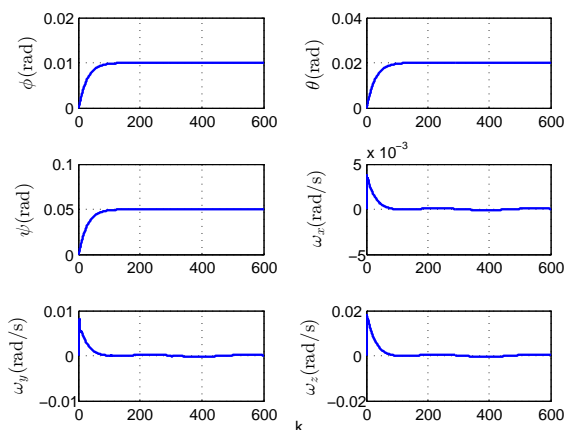

Fig. 3. Fault-tolerant control result in a time-varying actuator fault scenario

\section{CONCLUSIONS}

In this paper a novel self-healing attitude tracking control method based on simultaneous fault estimation and control design for satellite is proposed. The main idea of the proposed self-healing method is to construct an augmented error system and then design a fault estimator/controller based on the augmented system. In this paper, the design of the fault estimator/controller is formulated as a multi-objective $H_{\infty}$ optimization problem and further transformed as a set of LMIs. Finally, numerical simulations are used to demonstrate the performance of the proposed method.

\section{REFERENCES}

[1] B. Xiao, Q. L. Hu and M. I. Friswell, Active fault-tolerant attitude control for flexible spacecraft with loss of actuator effectiveness, International Journal of Adaptive Control and Signal Processing, vol. 27, no. 11, pp. 925-943, 2013.

[2] Y. J. Ma, B. Jiang and G. Tao, Adaptive actuator failure identification for microsatellites under closed-loop control, IEEE Transactions on Control Systems Technology, vol. 23, no. 3, pp. 910-923, 2015.

[3] Y. Shen, Z. H. Wang and X. L. Zhang, Fault diagnosis and faulttolerant control for sampled-data attitude control systems: An indirect approach, Proceedings of the Institution of Mechanical Engineers, Part G: Journal of Aerospace Engineering, vol. 228, pp. 1047-1057, 2014.

[4] A. Chamseddine, C. Join and D. Theilliol, Trajectory planning/replanning for satellite systems in rendezvous mission in the presence of actuator faults based on attainable efforts analysis, International Journal of Systems Science, vol. 46, no. 4, pp. 690-701, 2015.

[5] D. Bustan, N. Pariz and S. K. H. Sani, Robust fault-tolerant tracking control design for spacecraft under control input saturation, ISA Transactions, vol. 53, no. 4, pp. 1073-1080, 2014.

[6] L. J. Liu, Y. Shen and E. H. Dowell, Integrated adaptive fault-tolerant $H_{\infty}$ output feedback control with adaptive fault identification, Journal of Guidance, Control, and Dynamics, vol. 35, no. 3, pp. 881-889, 2012.

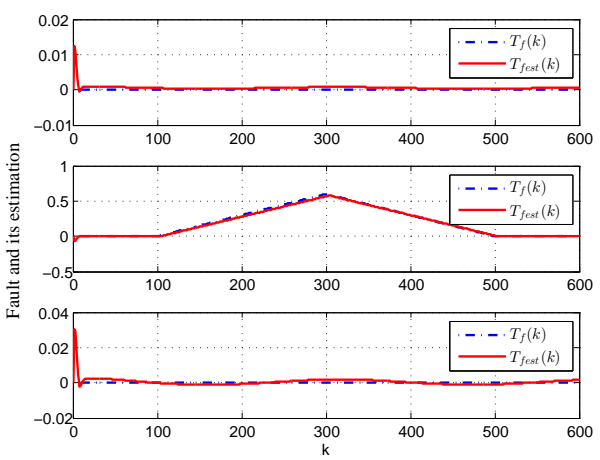

Fig. 4. Fault estimation result in a time-varying actuator fault scenario

[7] Y. Shen, L. J. Liu and E. H. Dowell, Adaptive fault-tolerant robust control for a linear system with adaptive fault identification, IET Control Theory \& Applications, vol. 7, no. 2, pp. 246-252, 2013.

[8] A. Casavola, M. Rodrigues and D. Theilliol, Self-healing control architectures and design methodologies for linear parameter varying systems, International journal of robust and nonlinear control, vol. 25, no. 5, pp. 625-626, 2015.

[9] X. Qi, D. Theilliol, J. T. Qi, Y. M. Zhang, and J. Han, Self healing control method against unmanned helicopter actuator stuck faults, International conference on Unmanned Aircraft System, Orlando, 2014, pp. 842-847.

[10] H. Wang and G. H. Yang, Simultaneous fault detection and control for uncertain linear discrete-time systems, IET Control Theory \& Applications, vol. 3, no. 5, pp. 583-594. 2009.

[11] M. J. Khosrowjerdi, R. Nikoukhah and N. Safari-Shad, A mixed $H_{2} / H_{\infty}$ approach to simultaneous fault detection and control, Automatica, vol. 40, no. 2, pp. 261-267, 2004.

[12] M. R. Davoodi, A. Golabi, H. A. Talebi and H. R. Momeni, Simultaneous fault detection and control design for switched linear systems based on dynamic observer, Optimal Control Applications and Methods, vol. 34, no. 1, pp. 35-52, 2013.

[13] A. Marcos and G. J. Balas, A robust integrated controller/diagnosis aircraft application, International Journal of Robust and Nonlinear Control, vol. 15, no. 12, pp. 531-551, 2005.

[14] W. J. Liu, Y. Chen and M. L. Ni, An linear matrix inequality approach to simultaneous fault detection and control design for LTI systems, Proceedings of the 33rd Chinese Control Conference, Nanjing, China, 2014, pp. 3249-3254.

[15] S. X. Ding, Integrated design of feedback controllers and fault detectors, Annual Reviews in Control, vol. 33, no. 2, pp. 124-135, 2009.

[16] J. L. Lan, R. J. Patton, a new strategy for integration of fault estimation within fault-tolerant control, Automatic, vol. 69, pp. 48-59, 2016.

[17] Y. Shen, Z. H. Wang, X. L. Zhang, Fault diagnosis and fault tolerant control for sampled-data attitude control systems: an indirect approach, Proceeding of The Institution of Mechanical Engineers, Part-G-Journal of Aerospace Engineering, vol. 228, no. 7, pp. 1047-1057, 2014.

[18] C. D. Yang and Y. P. Sun, Mixed $H_{2} / H_{\infty}$ state-feedback design for microsatellite attitude control, Control Engineering Practice, vol. 10, no. 9, pp. 951-970, 2002.

[19] J. Park, G. Rizzoni, W. Ribbens, On the representation of sensor faults in fault detection filters, Automatica, vol. 30, no. 11, pp. 1793-1795, 1994.

[20] M. Rodrigues, M. Sahnoun, D. Theilliol and J. C. Ponsart, Sensor fault detection and isolation filter for polytopic LPV systems: A winding machine application, Journal of Process Control, vol. 23, pp. 805-816, 2013.

[21] P. Gahinet and P. Apkarian, A linear matrix inequality approach to $H_{\infty}$ control, International Journal of Robust and Nonlinear Control, vol. 4, no. 4, pp. 421-448, 1994.

[22] S. Boyd, L. Ghaoui, E. Feron and V. Balakrishnan, Linear Matrix Inequalities in System and Control Theory, Society for Industrial and Applied Mathematics (SIAM), 1994.

[23] Y. Tao, D. Shen, M. Q. Fang, Y. Q. Wang, Reliable $H_{\infty}$ control of discrete-time systems against random intermittent faults, International Journal of Systems Science, 2014: 1-11. 